\title{
USING THE WI-FI TECHNOLOGY AND DEVICES TO TRANSMIT RESULTS OF HUMAN BODY BIOSIGNALS PROCESSING
}

\author{
Xakimjon N. Zaynidinov ${ }^{1}$, Azambek A. Turakulov' ${ }^{2}$ Fotima T. Mullajonova ${ }^{3}$ \\ ${ }^{1}$ The Tashkent University of Information Technologies, Tashkent, Uzbekistan \\ ${ }^{2,3}$ The Namangan institute of Engineering and Technology, Namangan, Uzbekistan
}

\begin{abstract}
The automated temperature monitoring device provides data transmission to external computing systems, including a computer. This restriction requires the direct presence of external devices near the person whose temperature is being measured. Since the automated device is portable, which can be used in home, outdoor and camping conditions, as well as the objects of measurement can be children, the elderly and people with limited selfmovement abilities, the aforementioned disadvantage created additional inconveniences. This article discusses the problems of integrating a Wi-Fi device to an automated temperature monitoring device in order to organize wireless data exchange in external devices.
\end{abstract}

Keywords: LCD1602 liquid crystal display, I2C protocol, Wi-Fi, ESP8266 family, ESP-12f module, microcontrollers.

\section{INTRODUCTION}

In today's pandemic conditions, monitoring people's health is one of the urgent problems. The very first symptom of COVID-19 is a slight increase in human body temperature. Timely detection of temperature makes it possible to identify a sick person at an early stage of the disease.

In order to facilitate systematic monitoring of human body temperature, we (the authors of the article) have created a device for simultaneous measurement of temperatures on different parts of the body and a system for automated digital data processing for visualization and preservation of results [1-3]. This device allows you to simultaneously measure temperatures in up to 127 parts of the human body using DS18B20 sensors connected to a single cable with three veins. A bus and the 1-Wire protocol are used to transmit data to the microcontroller via a single cable [4]. To visualize the measurement results and data processing, the LCD1602 liquid crystal display is integrated into the device. The signals from the microcontroller to the display are transmitted via the device and the $\mathrm{I} 2 \mathrm{C}$ protocol.

The automated temperature monitoring device provides data transmission to external computing systems, including a computer. At the same time, a serial USB port is used. This restriction requires the direct presence of external devices near the person whose temperature is being measured. Since the automated device is portable, which can be used in home, outdoor and camping conditions, as well as the objects of measurement can be children, the elderly and people with limited self-movement abilities, the aforementioned disadvantage created additional inconveniences. As a result, there was a need for wireless remote data transmission in order to observe the results of monitoring at a distance by parents or other close people.

This article discusses the problems of integrating a Wi-Fi device to an automated temperature monitoring device in order to organize wireless data exchange in external devices.

\section{CHOOSING THE APPROPRIATE WI-FI DEVICE MODEL}

Since the temperature monitoring device is designed for a wide range of people, when developing it, the main parameter was the availability of its parts at cost and the ease of its use by people who do not have special education and skills. Therefore, the Arduino Nano microcontroller was used in the device, which has a small size and good computing capabilities. Therefore, for a Wi-Fi device, size, cost and capabilities remain the main requirements.

Currently, there are a huge number of models of Wi-Fi devices compatible with the Arduino Nano microcontroller. For our case, the most suitable are Wi-Fi devices of the ESP8266 family. The ESP8266 chip is popular, publicly available and with the default software download for working with commands, which is enough to work with the Arduino Nano microcontroller. Another advantage of the ESP8266 chip is its low price. 


\section{International Advanced Research Journal in Science, Engineering and Technology}

Vol. 8, Issue 10, October 2021

DOI: 10.17148/IARJSET.2021.81001

On the basis of this chip, there are many ready-made Wi-Fi modules for working with microcontrollers. The images of the most popular among them are shown in Figure 1.

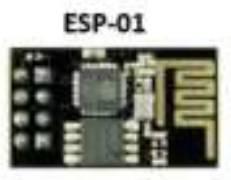

ESP-06

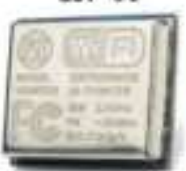

ESP-11

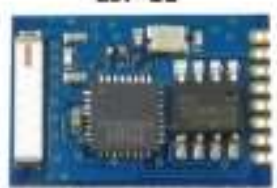

ESP-02

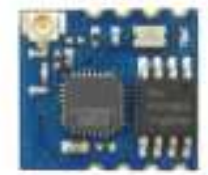

ESP.07
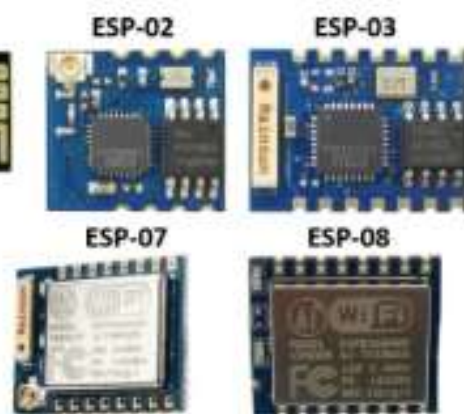

ESP-08

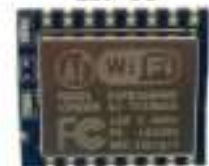

ESP-12

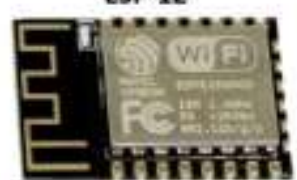

ESP-13
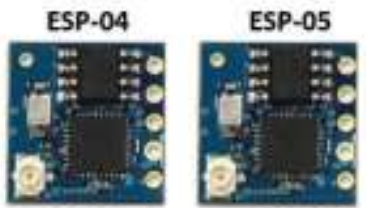

ESP-09
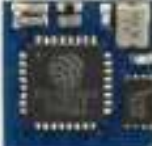

ESP-10

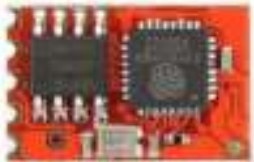

ESP-14
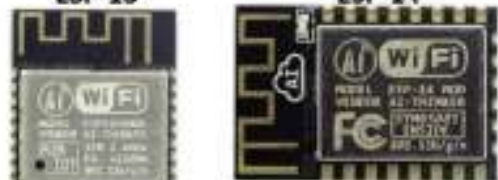

Fig.1. Popular models of Wi-Fi modules based on the ESP8266 chip.

In addition, each module shown in this figure has several modifications. Including the ESP-12 series of devices has modifications Without violating the generality, among these modules we choose the ESP-12f module (Fig.2), which is most suitable for our case.

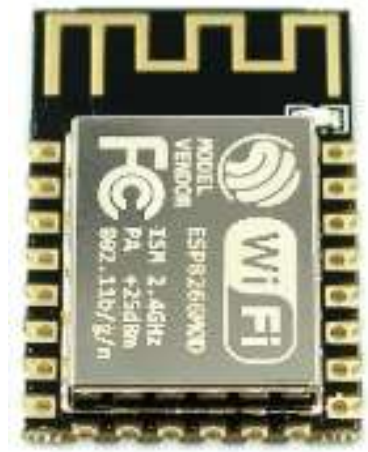

Fig.2. A ready-made Wi-Fi module compatible with the Arduino Nano microcontroller.

Despite the miniature size - $16 \times 24 \times 3 \mathrm{~mm}$, the module has impressive characteristics $\bullet$

- $4 \mathrm{MB}$ of internal flash memory;

- $\quad$ Serial UART interface: 2 outputs ( $\mathrm{Rx}$ and $\mathrm{Tx}) \bullet$

- SPI interface

- $\quad$ Ready for FCC certification (metal screen);

- $\quad 17$ GPIO pins, Chip enable pin, ADC pin, Reset pin;

- Built-in PCB antenna with improved performance:

- $\quad 2.4 \mathrm{GHz}$ radio unit complies with $802.11 \mathrm{~b} / \mathrm{g} / \mathrm{n}$;

- $\quad$ Wi-Fi Direct (P2P), soft-AP (access point) modes are supported;

- $\quad$ built-in TCP/IP stack;

- built-in radio components, direct connection to the antenna (TR switch, balun, LNA, power amplifier and matching circuits inside the chip);

- $\quad$ output power $+19.5 \mathrm{dBm}$ in $802.11 \mathrm{~b}$ mode;

- 32-bit RISC core processor;

- $\quad$ WEP, TKIP, AES, and WAPI encryption (including WPA2 supported).

The ESP-12f module has 22 pins (Fig. 3), the purpose of which is given below. 
DOI: 10.17148/IARJSET.2021.81001

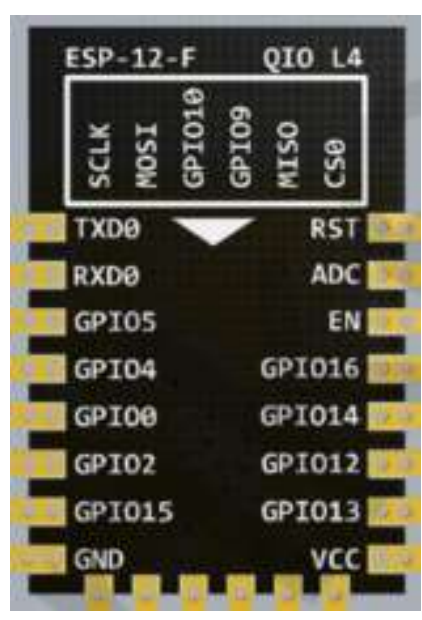

Figure 3. Layout of WiFi ESP-12f pins.

- GPIO0-GPIO16 - general purpose contacts, data input/output. Some of them can be reassigned to other functions, which will be listed below;

- $\quad$ TXD (GPIO1) - pin for transmitting data over the UART bus;

- $\quad$ RXD0 (GPIO3) - pin for receiving data over the UART bus;

- $\quad$ RST (EXT_RSTB, RESET) - module restart pin, active with a low-level signal;

- $\quad$ ADC (TOUT) - analog-to-digital converter (ADC). The permissible input voltage is 0-3.3 V, the range of converted digital values in the range 0-1023;

- $\quad$ GND - common grounding;

- $\quad \mathrm{VC}$ - contact is combined with the output of the input voltage of 5 volts;

- $\quad$ EN - pin for activation and passivation of the device;

- $\quad$ SCLK (GPIO6) - pin for clocking when working in I2C mode;

- $\quad$ MOSI (GPIO8) - pin for setting the mode "master output, slave input";

- $\quad$ MISO (GPIO7) - pin for setting the "master input, slave output" mode;

- $\quad$ CS0 (GPIO11) - pin for chip selection.

It should be noted that along with its capabilities, the ESP-12f module has some inconveniences. One of them is a problem with the power supply. It appears when using the module together with the Arduino Nano microcontroller. The ESP-12f module requires electricity with a voltage of $3.3 \mathrm{~V}$ and consumes about $300 \mathrm{~mA} / \mathrm{h}$ of current. And the total output current of the microcontroller is no more than $200 \mathrm{~mA} / \mathrm{h}$, i.e. it cannot provide the Wi-Fi module with electricity. An additional energy source is required. In order not to increase the size of the body temperature monitoring device, it would be advisable to use the energy source of the microcontroller. But the voltage of this source is $5 \mathrm{~V}$ (when using the USB port) or from 7 to $10 \mathrm{~V}$ (via the pin VIN of the microcontroller). Such voltages are not allowed for ESP$12 \mathrm{f}$, they can lead to the burning of the module.

To solve this problem, we can use a 3.3V power supply module based on the AMS1117-3.3V chip (see Figure 4). It can convert the input voltage from 4.4 to $7 \mathrm{~V}$ to a stable $3.3 \mathrm{~V}$ with a current of up to $800 \mathrm{~mA}$, which is optimal for ESP-12F.
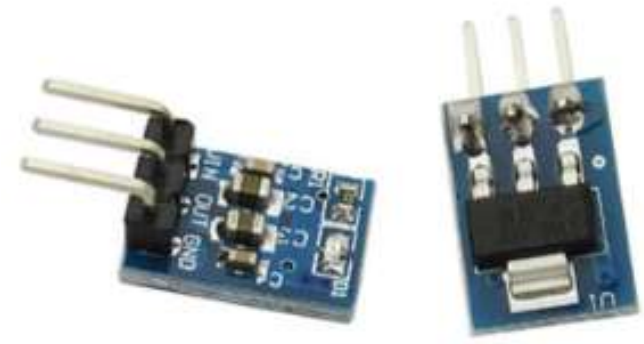

Figure 4. 3.3V power supply module based on the AMS1117-3.3V chip.

The module has 3 pins: GND - common ground, IN - for input voltage, OUT - for converted stable voltage 3.3V. The size of the module $25 \times 11 \mathrm{~mm}$ allows you to place it inside the temperature monitoring unit without increasing its size. Now let's give a diagram of the connection of the ESL-12f module to the Arduino Nano microcontroller (see Figure 5) as a set-top box for exchanging information with external devices using Wi-Fi. 


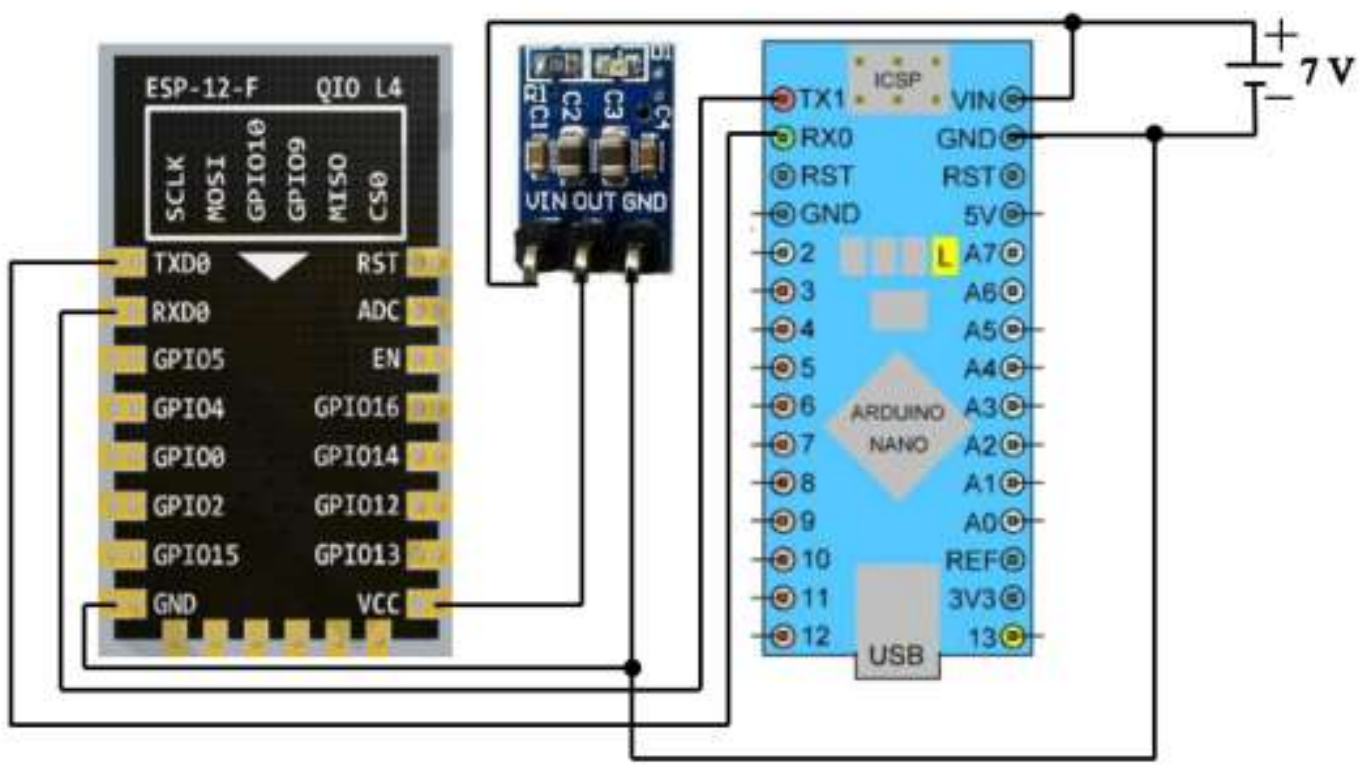

Figure 5. Connection diagram of the ESP-12f module to the Arduino Nano microcontroller

It should be emphasized here that when connecting the pin of the microcontroller TX connects to the pin of the Wi-Fi module DX and vice versa, the pin DX with the pin TX, respectively.

\section{PROGRAMMING THE ESP-12F MODULE USING THE ARDUINO IDE}

Manufacturers have loaded a default program into the module's memory to work only with AT commands. This is enough to organize the exchange of information with external devices using the ESP-12f module. In this case, the module only plays the role of a prefix for transmitting and receiving data. The computing capabilities of the module are not used, which makes it possible to save the energy resource of a portable body temperature monitoring device. But if necessary, you can reprogram the ESP-12f module to change the necessary parameters. As a programmer, you can use the Arduino IDE.

To work with ESP8266-based boards, we must first install the appropriate driver. It can be found on the Internet sites of device manufacturers [6]. Uploading the archive file esp8266-3.0.2.zip and put it in the working folder.

Next, you need to unpack the archive into the service folder of the Arduino IDE environment. In our example, this folder has the address C:IProgram Files (x86)\Arduinolhardwarelesp8266\ ( fig.6).

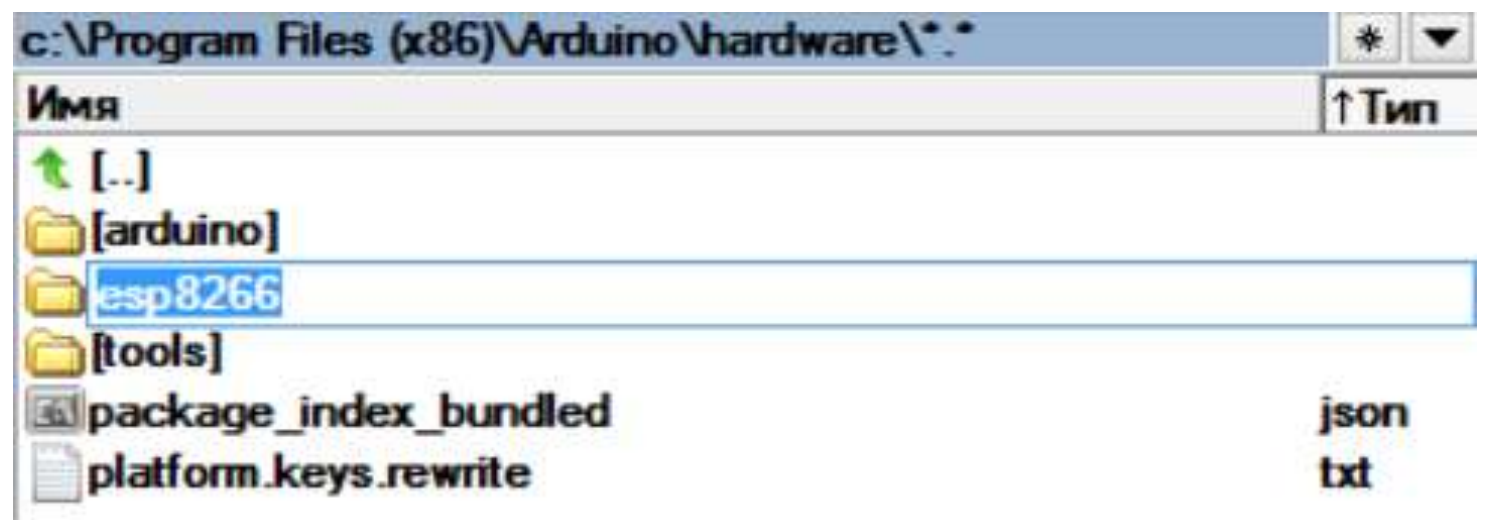

Figure 6. Folder for hosting the driver for the ESP8266 device family.

Then we launch the Arduino IDE environment and specify "Generic ESP8266 Module" as the board in the "Tools" menu section ( Fig.7). 
Vol. 8, Issue 10, October 2021

DOI: 10.17148/IARJSET.2021.81001

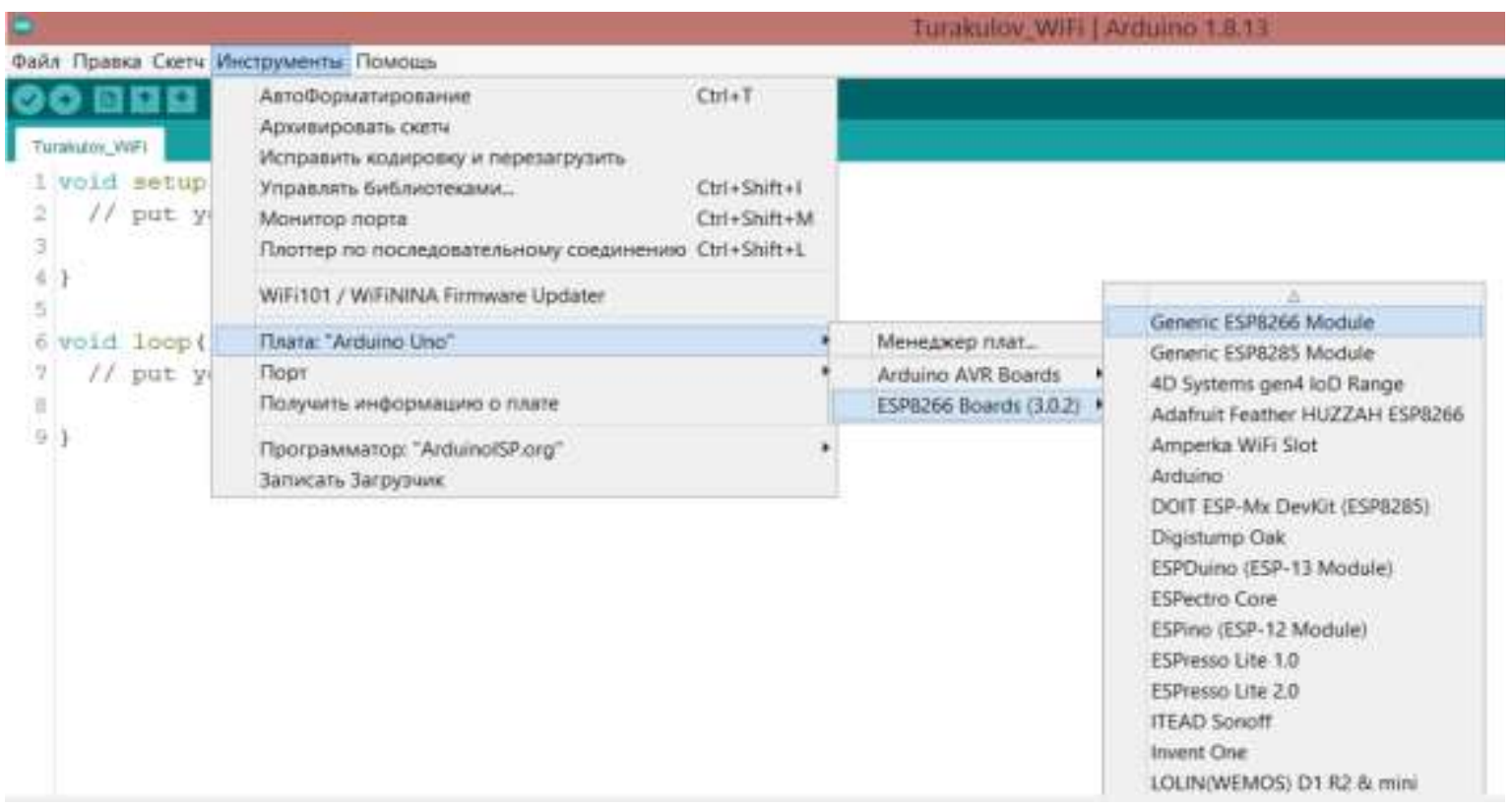

Figure 7. Installing the Generic ESP8266 Module board as a working board of the Arduino IDE environment.

Now, in the "Tools" menu section, lines appear for configuring the ESP8266 Wi-Fi module (see Figure 8) where you can select the necessary parameters.

As mentioned above, we have given the possibility of reprogramming for cases of changing the default program or using the computing resources of the Wi-Fi module for information processing. And in our case, we are quite satisfied with the program downloaded by manufacturers to work with teams [8].

WiFi101 / WiFiNINA Firmware Updater
Плата: "Generic ESP8266 Module"
Builtin Led: "2"
Upload Speed: "115200"
CPU Frequency: "80 MHz"
Crystal Frequency: "26 MHz"
Flash Size: "1MB (FS:64KB OTA: 470KB)"
Flash Mode: "DOUT (compatible)"
Flash Frequency: "40MHz"
Reset Method: "dtr (aka nodemcu)"
Debug port: "Disabled"
Debug Level: "Hичero"
IwIP Variant: "v2 Lower Memory"
VTables: "Flash"
C++ Exceptions: "Disabled (new aborts on oom)"
Stack Protection: "Disabled"
Erase Flash: "Only Sketch"
Espressif FW: "nonos-sdk 2.2.1+100 (190703)"
SSL Support: "All SSL ciphers (most compatible)"
MMU: "32KB cache + 32KB IRAM (balanced)"
Non-32-Bit Access: "Use pgm_read macros for IRAM/PROGMEM"
Порт
Получить информацию о плате
Программатор
Записать 3arрyзчик

Figure 8. Arduino IDE menu bars for selecting Wi-Fi module parameters. 


\section{International Advanced Research Journal in Science, Engineering and Technology}

Vol. 8, Issue 10, October 2021

\section{DOI: $10.17148 /$ IARJSET.2021.81001}

\section{CONCLUSION}

Based on the above studies, the following conclusions can be drawn:

When transmitting data from body temperature sensors to a microcontroller, it is advisable to use a cable with three veins using a bus and a 1-Wire protocol. This architecture allows us to measure and transmit heat signals simultaneously to 127 body parts.

For digital signal processing, it is recommended to use a miniature Arduino Nano microcontroller, so that the size of a portable human body temperature monitoring device does not increase.

In order for the proposed device to be used by a wide range of people, the results obtained after digital processing of biosignals must necessarily be visualized in the device itself. A miniature LCD1602 monitor is recommended for displaying temperatures of various parts of the body in the form of numbers. When transferring data from the microcontroller to the monitor, it is advisable to use the device and the $\mathrm{I} 2 \mathrm{C}$ protocol to minimize the number of occupied pins.

For long-term preservation and graphical visualization of the results, it is proposed to use external devices such as a computer, laptop, mobile phone, etc. For wireless data transmission, it is recommended to use the ESP-12f Wi-Fi device based on the ESP8266 processor, which is ideal for both small size and large capabilities. For more clarity, it is recommended to create a user interface using a SCADA system [5].

\section{REFERENCES}

[1]. Azambek Turakulov, Fotima Mullajonova. Automated system for monitoring the body temperature of children, disabled and bedridden people using continuous analysis. DIAGNOSTICS, 2020, VOLUME 21, NO. 3. ISSN 1641-6414. E-ISSN $2449-5220$. https://doi.org/10.29354/diag/125314

[2]. Azambek Turakulov, Fotima Mullajonova. Device and software for automated processing of human body data. Scientific and Technical Journal of Namangan Institute of Engineering and Technology. Volume 4, 2019, 152-163 pages.

[3]. A.Turakulov, F.Mullajonova. An algorithm for automated processing of thermal signals of the human body. // In the journal "Development of Science and Technology" of the Bukhara Institute of Engineering and Technology. No. 5, 2018.

[4]. A.A.Turakulov, F.T.Mullajonova. The use of a 1-wire protocol in a multi-sensor biosignal monitoring system. Materials of the scientific conference. Namangan Institute of Engineering and Technology. Namangan, 2021, July 24-25.

[5]. Mullajonova F. The possibility of creating a user interface for monitoring biosignals using the Simplified Scada system. Materials at the VIII International Conference "Science and Education in the Modern World: Challenges of the XXI century". Nur-Sultan, Kazakhstan, April 2012.

[6]. https://www.compel.ru/lib/123539

[7]. https://sourceforge.net/projects/esp8266-arduino-core.mirror/files/2.4.2/

[8]. http://espressif.com/ 